\title{
Mudanças comportamentais da população da cidade de Rio Grande (RS) frente à
}

\section{pandemia}

\author{
Behavioral changes of the population in the city of Rio Grande (RS) facing the pandemic \\ Cambios de comportamiento de la población de la ciudad de Río Grande (RS) frente a la pandemia
}

Recebido: 27/01/2021 | Revisado: 02/02/2021 | Aceito: 08/02/2021 | Publicado: 15/02/2021

\author{
Lara Beatriz Ferreira \\ ORCID: https://orcid.org/0000-0001-6932-8395 \\ Universidade Federal do Rio Grande, Brasil \\ E-mail: lara.beatriz.ferreira@hotmail.com \\ Matheus Ferreira Lasmar \\ ORCID: https://orcid.org/0000-0002-8585-8799 \\ Universidade Federal do Rio Grande, Brasil \\ E-mail: matheusflasmar@hotmail.com \\ Daniela Fernandes Ramos \\ ORCID: https://orcid.org/0000-000168889553 \\ Universidade Federal do Rio Grande, Brasil \\ E-mail: daniferamos@gmail.com
}

\begin{abstract}
Resumo
Classificada como pandemia pela Organização mundial da saúde, a COVID-19 tornou-se protagonista quanto às demandas de saúde pública ao redor do mundo. No Brasil, segundo país com mais mortes pela doença, o Rio Grande do Sul é o sexto estado mais afetado pelo novo coronavírus. Assim, realizou-se uma pesquisa online com os moradores da cidade de Rio Grande, no sul do estado, para avaliar o impacto da pandemia sobre a população local em relação aos hábitos comportamentais de diferentes grupos. Através de um questionário online foi possível coletar dados de uma amostra de 1188 indivíduos e subsidiar um trabalho quantitativo e descritivo acerca das mudanças comportamentais dos rio-grandinos frente à COVID-19. Os resultados mostraram que apesar de os homens terem sido aqueles que mais mudaram hábitos comportamentais com a chegada da pandemia - como a lavagem frequente das mãos -, as mulheres foram as que tiveram os maiores percentuais de distanciamento social e apresentaram maiores preocupações quanto a serem infectadas. Essa tendência comportamental se reflete nos dados epidemiológicos da cidade, em que mulheres foram minoria em relação ao número de casos e, também, número de óbitos no município. Fatores como renda e escolaridade também influenciam o comportamento dos rio-grandinos em relação aos hábitos durante a pandemia. Dessa forma, o estudo e a compreensão das práticas adotadas pela população é fundamental para que se aperfeiçoem as campanhas preventivas e haja uma readequação no diálogo com a população para aprimorar as medidas de contenção da pandemia.
\end{abstract}

Palavras-chave: Covid-19; Pandemia; Prevenção; Rio Grande do Sul; Comportamento social.

\begin{abstract}
Classified as a pandemic by the World Health Organization, COVID-19 has become a protagonist in public health demands around the world. In Brazil, the second country with the most deaths, Rio Grande do Sul is the sixth state most affected by COVID-19. Therefore, an online research was conducted with the residents of the city of Rio Grande, in the south of the state, to assess the impact of the pandemic on the local population in relation to behavioral habits of different groups. Through an online questionnaire, it was possible to collect data from 1188 individuals and to support a quantitative and descriptive study about the behavioral changes of the rio-grandinos in relation to COVID-19. The results showed that although men were the ones who changed the most behavioral habits with the arrival of the pandemic - such as frequent hand washing - women were the ones who had the highest percentages of social distance and had the highest concerns about being infected. This behavioral trend is reflected in the city's epidemiological data, in which women were in the minority in relation to the number of cases and also the number of deaths in the city. Factors such as family income and schooling influence the behavior of rio-grandinos in relation to habits during the pandemic. As such, the study and understanding of practices adopted by the population is fundamental to improve preventive campaigns and there is a readjustment in the dialogue with the population to improve measures to contain the pandemic.
\end{abstract}

Keywords: Covid-19; Pandemic; Prevention; Rio Grande do Sul; Social behavior. 


\begin{abstract}
Resumen
Clasificado como pandemia por la Organización Mundial de la Salud, el COVID-19 se ha convertido en protagonista de las demandas de salud pública en todo el mundo. En Brasil, el segundo país con más muertes por la enfermedad, Rio Grande do Sul es el sexto estado más afectado por el nuevo coronavirus. Así, se realizó una encuesta en línea con los residentes de la ciudad de Río Grande, en el sur del estado, para evaluar el impacto de la pandemia en la población local en relación con los hábitos de comportamiento de los diferentes grupos. A través de un cuestionario online fue posible recoger datos de una muestra de 1188 individuos y apoyar un trabajo cuantitativo y descriptivo sobre los cambios de comportamiento de los riojanos en relación con el COVID-19. Los resultados mostraron que, aunque los hombres fueron los que más cambiaron sus hábitos de comportamiento con la llegada de la pandemia -como lavarse las manos con frecuencia-, las mujeres fueron las que presentaron mayores porcentajes de distanciamiento social y tuvieron mayor preocupación por ser infectadas. Esta tendencia de comportamiento se refleja en los datos epidemiológicos de la ciudad, donde las mujeres eran minoría en relación con el número de casos y también con el número de muertes en el municipio. Factores como los ingresos y la escolaridad influyen en el comportamiento de los rio-grandinos en relación con los hábitos durante la pandemia. Así, el estudio y la comprensión de las prácticas adoptadas por la población es fundamental para mejorar las campañas preventivas y se produce un reajuste en el diálogo con la población para mejorar las medidas de contención de la pandemia.
\end{abstract}

Palabras clave: Covid-19; Pandemia; Prevención; Rio Grande do Sul; Comportamiento social.

\title{
1. Introdução
}

Em dezembro de 2019, em Wuhan, China, surgiu um novo tipo de infecção viral com apresentação clínica similar à pneumonia (Shereen, et al., 2020). Em 31 de dezembro, a Word Health Organization (WHO) foi alertada sobre os vários novos casos da infecção que foi atribuída ao vírus SARS-CoV-2, responsável pela doença COVID-19. A nova infecção se espalhou por diversos países até que, em 11 de março de 2020, a doença foi classificada como uma pandemia pela WHO e pela Organização Pan-Americana da Saúde (World Health Organization [WHO], 2020b, Organização Pan-Americana da Saúde [OPAS], 2020).

No Brasil, o primeiro caso foi registrado em 26 de fevereiro de 2020, na cidade de São Paulo, e, desde então, a COVID-19 tornou-se protagonista em relação às demandas de saúde pública em todo país (Rodriguez-Morales, et al., 2020). Segundo a Secretaria da Saúde do Estado do Rio Grande Do Sul (SES-RS), o estado do Rio Grande do Sul, até o dia 13 de janeiro de 2021, registrou 490.980 casos confirmados e 9.699 óbitos e se encontra na sexta posição no ranking dos estados do Brasil com maior número de casos confirmados. Já o município de Rio Grande, registrou 8.935 casos confirmados e 186 óbitos, ocupando a $11^{\circ}$ no ranking das cidades do estado com maior número de casos confirmados (Secretaria Estadual da Saúde do Estado do Rio Grande do Sul [SES-RS], 2021).

Desde a descoberta da nova doença até o atual momento, as pesquisas buscam o desenvolvimento ou remanejo de compostos farmacológicos capazes de combater a doença de maneira eficaz, além do esforço conjunto entre os países para o desenvolvimento de vacinas seguras e eficazes (Ferreira \& Ramos, 2020; Guimarães, 2020). No entanto, frente ao cenário de incertezas, a promoção de ações de controle e prevenção da infecção se fazem essenciais de maneira constante, já que a transmissão do vírus de pessoa para pessoa, ocorre principalmente por gotículas espalhadas, em caso de tosse ou espirro, e por contato direto com indivíduo infectado (Rothan \& Byrareddy, 2020). Diante disso, desde 19 de março de 2020, o governo do Estado do Rio Grande do Sul e a Prefeitura de Rio Grande, implementam protocolos e orientações de prevenção ao contágio pelo COVID-19, que corroboram com as orientações mundiais (SES-RG, 2020; Rio Grande - RS, 2020; Organização Mundial da Saúde [OMS], 2020; WHO, 2020a). Nesse sentido, tais orientações, segundo Rothan \& Byrareddy (2020), incluem medidas de proteção individual, como a lavagem frequente das mãos, uso de álcool gel e de máscara facial, além de isolamento social, sempre que possível. Sabe-se que as mãos possuem alta capacidade de abrigar microrganismos, constituindo-se, então, uma via importante de transmissão viral, nesse contexto a higienização das mãos pode ser uma medida eficaz para o enfretamento de doenças infectocontagiosas, incluindo a COVID-19 (Mbakaya, et al., 2017; Cowling, et al., 2009; West, et al., 2020). Além 
disso, é sabido que a "Zona T", referente aos olhos, nariz e boca, é a principal via de entrada viral, uma vez que o SARS-CoV2 pode entrar em contato direto com as mucosas e alcançar o tecido pulmonar, dessa forma, as máscaras faciais criam uma barreira física para propagação de gotículas e de manipulação constante dessa zona (West, et al., 2020; Pestana, et al., 2020).

Ademais, houve orientações em relação ao distanciamento social, ou seja, os indivíduos devem se restringir quanto às interações entre as pessoas em uma comunidade, evitando locais em que haja aglomerações. Essas medidas foram difundidas popularmente como isolamento social ou quarentena, por isso o termo isolamento social será utilizado nesse trabalho como sinônimo de distanciamento social (Aquino, et al., 2020). Diante dos estudos que abordaram esse tema, o isolamento social demonstra ser útil, porém não deve ser uma intervenção isolada, ou seja, devem ser realizadas em conjunto com orientações higiênicas, proteção individual e isolamento de pacientes infectados e doentes, garantindo uma melhor eficácia na diminuição da transmissão do vírus (Nussbaumer-Streit, et al., 2020; Ferguson, et al., 2020).

Assim, o comportamento comunitário é crucial para evitar o crescimento do número de casos e óbitos pela COVID19, portanto, é imprescindível conhecer o comportamento humano frente a atual pandemia. Nesse contexto, o objetivo desse trabalho é analisar diferentes perfis socioeconômicos dos moradores da cidade de Rio Grande (RS) que mudaram seus hábitos durante a pandemia a fim de que, as informações fornecidas por esse trabalho possam contribuir para a otimização de estratégias públicas já estabelecidas, uma vez que possibilitam abordagens mais direcionadas em diferentes grupos sociais, aumentando a adesão da população às medidas de controle e prevenção à COVID-19.

\section{Metodologia}

Nesse contexto, o campo de ação foi na cidade de Rio Grande (Rio Grande do Sul, Brasil), cidade costeira com extensão de 2.709,391 km², população de 197.228 indivíduos sendo que, de acordo com o último senso realizado em 2010, $29,4 \%$ dos moradores tinham até 19 anos, $17,1 \%$ de 20 a 29 anos, 27,6\% entre 30 e 49 anos, 19,9\% entre 50 e 69 anos e 6,5\% acima de 69 anos. Em relação à economia, a cidade tem seu Produto Interno Bruno (PIB) de aproximadamente 51.681, além de o salário médio mensal dos trabalhadores formais ser referente a 3,6 salários mínimos e um Índice de Desenvolvimento Humano de 0,744, segundo dados fornecidos pelo Senso Demográfico feito pelo Instituto Brasileiro de Geografia e Estatística (Instituto Brasileiro de Geografia e Estatística, [IBGE], 2010 b).

Nesse sentido, o estudo em questão segue uma metodologia quantitativa e descritiva, delineamento embasado de acordo com Pereira, et al. (2018). Para isso foi elaborado um questionário online por meio da ferramenta Google Forms ${ }^{\circledR}$ de forma que, de maneira fácil e rápida, alcançasse o maior número de habitantes da cidade de Rio Grande-RS. Para delimitar o erro amostral, realizou-se uma estimativa do acesso à rede pelos rio-grandinos. Para isso, foi utilizado como base referencial a "Pesquisa sobre o uso das Tecnologias de Informação e Comunicação nos domicílios brasileiros" realizada em 2018, que compreende ao acesso à internet pela população na região sul do país. Nesse contexto, foi verificado que $75 \%$ da população residente no sul do Brasil possui acesso à internet, assim, estabeleceu-se que 147.921 pessoas têm acesso à internet na cidade de Rio Grande. Dessa forma, de acordo com os cálculos realizados por meio da plataforma SurveyMonkey ${ }^{\circledR}$, o erro amostral desse estudo é de 3\% e o grau de confiança de $95 \%$ com N amostral de 1.188 .

O formulário foi divulgado e difundido nos meios de comunicação midiática no dia 31 de maio de 2020 e ficou disponível para preenchimento durante um mês, esse período foi o escolhido pois no mês de maio, segundo o Modelo de Distanciamento Controlado do Rio Grande do Sul, a região encontrava-se em bandeira laranja, ou seja, em um grau de risco médio. As variáveis investigadas foram divididas em sessões para melhor compreensão dos participantes e organização do questionário, porém, somente teriam acesso às perguntas, os participantes que se autodeclarassem maiores de 18 anos de idade. 
As frentes foram elaboradas de forma que abordassem o perfil do entrevistado (de forma confidencial), contexto socioeconômico e hábitos de vida. Primeiramente, foram realizadas perguntas pessoais como: sexo, idade, escolaridade e renda. Por conseguinte, as duas últimas sessões tiveram como objetivo avaliar as mudanças no comportamento higiênico dos habitantes frente à pandemia e o medo em infectar-se. Dessa forma, as sessões foram divididas em dois períodos distintos (antes da pandemia e durante a pandemia). As variáveis investigadas foram relacionadas à higienização das mãos, uso de máscara de proteção, além de uma escala numérica crescente (variando de 0 a 5) referente ao medo dos indivíduos em serem infectados com o novo coronavírus. Nessa escala numérica, zero é equivalente à ausência de medo e cinco equivalente ao medo extremo de infectar-se.

Os dados obtidos foram organizados em planilhas de acordo com cada sessão por meio da ferramenta Microsoft Excel e, para análise e correlações, houve a determinação da frequência de cada variável para posterior organização em tabelas. Ademais, o projeto foi avaliado e aprovado pelo Comitê de Ética da Universidade Federal do Rio Grande - CEP/FURG sob o número CAAE 24643219.7.0000.5324. Por tratar-se de uma pesquisa de opinião via online, o termo de consentimento livre e esclarecido foi substituído por um texto prévio explicativo e a auto declaração do participante a maior idade.

\section{Resultados}

A fim de avaliar o comportamento dos rio-grandinos frente à pandemia, primeiramente foi realizado um levantamento sobre o perfil dos entrevistados, avaliando aspectos de classificação como sexo, idade, escolaridade e renda, conforme apresenta Tabela 1. 
Tabela 1 - Perfil socioeconômico dos entrevistados residentes na cidade de Rio Grande, Rio Grande do Sul, Brasil, desde o início da pandemia até o dia 30 de junho de 2020.

\begin{tabular}{lcc}
\hline Variáveis & $\mathbf{N}$ & \% \\
\hline Sexo & & \\
\hline Feminino & 915 & 77 \\
Masculino & 273 & 23 \\
\hline Faixa Etária (anos) & & \\
\hline 18-29 & 332 & 27,9 \\
30-49 & 469 & 39,5 \\
50-69 & 361 & 30,4 \\
$>$ 69 & 26 & 2,2 \\
\hline Escolaridade & & \\
\hline Ensino fundamental & 24 & 2 \\
Ensino médio & 152 & 12,8 \\
Graduação & 501 & 42,2 \\
Pós-graduação & 511 & 43 \\
\hline Renda & & \\
\hline Até 1 salário mínimo & 88 & 7,41 \\
De 1 a 3 salários mínimos & 314 & 26,43 \\
De 3 a 6 salários mínimos & 393 & 33,08 \\
De 6 a 9 salários mínimos & 164 & 13,8 \\
Mais de 9 salários mínimos & 214 & 18,01 \\
Nenhuma renda & 1.188 & 1,26 \\
\hline Total & & \\
\hline & & \\
\hline
\end{tabular}

Fonte: Autores (2020).

Como propriedades da amostra verificou-se que $77 \%(n=915)$ eram do sexo feminino, 39,5\% $(n=469)$, tinham entre 30 e 49 anos e a grande maioria, 85,2\% ( $=1012)$, apresentavam níveis de escolaridade altos (graduação ou pós-graduação). Ademais, 59,5\% $(\mathrm{n}=707)$ dos entrevistados apresentaram renda de 1 a 6 salários mínimos (entre $\mathrm{R} \$ 1.039$ e $\mathrm{R} \$ 6.234)$.

Em relação às mudanças de hábitos acerca de medidas de prevenção à COVID-19, foi observado um aumento no número de indivíduos que passaram a higienizar as mãos, de modo que, a lavagem frequente das mãos aumentou de forma considerável, conforme apresenta Tabela 2. 
Tabela 2 - Mudança de hábitos dos entrevistados residentes na cidade de Rio Grande, Rio Grande do Sul, Brasil, desde o início da pandemia até o dia 30 de junho de 2020.

\begin{tabular}{clccc}
\hline & & $\mathbf{N}$ & $\%$ \\
\hline \multirow{2}{*}{ Lavagem frequente das mãos (\%) } & Antes & 1035 & 87,1 \\
\cline { 2 - 2 } & Durante & 1176 & 98,9 \\
\hline \multirow{2}{*}{ Uso de álcool/álcool em gel (\%) } & Antes & 440 & 37 \\
\cline { 2 - 2 } & Durante & 1151 & 96,9 \\
\hline \multirow{2}{*}{ Uso de máscara de proteção (\%) } & Antes & 59 & 5 \\
\cline { 2 - 2 } & Durante & 1145 & 96,4 \\
\hline \multirow{2}{*}{ Isolamento social (\%) } & Sim & 1040 & 87,5 \\
\cline { 2 - 3 } & Não & 148 & 12,5 \\
\hline Total & & $\mathbf{1 . 1 8 8}$ & $\mathbf{1 0 0}$ \\
\hline
\end{tabular}

Fonte: Autores (2020).

Sobre a lavagens das mãos frequentemente, esse hábito passou de 87,1\% ( $\mathrm{n}=1035)$ para 98,9\% após o início da pandemia ( $\mathrm{n}=1176)$ e o uso de álcool/álcool em gel passou de 37\% ( $\mathrm{n}=440)$ para 96,89\% $(\mathrm{n}=1151)$. Para mais, a porcentagem de indivíduos que afirmaram usar máscara de proteção facial é de 96,4\% $(\mathrm{n}=1145)$, além de que, $87,5 \%(\mathrm{n}=$ 1040) referiam estar em isolamento social.

Após a análise geral dos dados obtidos, foram feitas algumas correlações entre as mudanças de hábitos e o medo de infectar-se com os dados socioeconômicos: sexo, faixa etária, escolaridade e renda, correlações apresentadas pela Tabela 3. 
Tabela 3 - Mudança de hábitos dos entrevistados residentes na cidade de Rio Grande, Rio Grande do Sul, Brasil, desde o início da pandemia até o dia 30 de junho de 2020, de acordo com o sexo, faixa etária, escolaridade e renda.

\begin{tabular}{|c|c|c|c|c|c|c|c|c|}
\hline & \multicolumn{2}{|c|}{$\begin{array}{l}\text { Lavagem frequente das } \\
\text { mãos }(\%)\end{array}$} & \multicolumn{2}{|c|}{$\begin{array}{c}\text { Uso de álcool/álcool em } \\
\text { gel }(\%)\end{array}$} & \multicolumn{2}{|c|}{$\begin{array}{l}\text { Uso de máscara de } \\
\text { proteção }(\%)\end{array}$} & \multicolumn{2}{|c|}{$\begin{array}{l}\text { Isolamento social } \\
(\%)\end{array}$} \\
\hline & Antes & Durante & Antes & Durante & Antes & Durante & Sim & Não \\
\hline \multicolumn{9}{|l|}{ Sexo } \\
\hline Feminino & 88,6 & 99 & 39,3 & 97,5 & 4,6 & 96,4 & 89,8 & 10,2 \\
\hline Masculino & 82,1 & 98,5 & 29,3 & 94,9 & 6,2 & 96,3 & 79,9 & 20,1 \\
\hline \multicolumn{9}{|l|}{ Faixa etária (anos) } \\
\hline $18-29$ & 82,5 & 98,8 & 38,3 & 95,8 & 5,7 & 95,8 & 91 & 9 \\
\hline $30-49$ & 88,1 & 98,7 & 39,4 & 96,4 & 4,1 & 95,9 & 82,7 & 17,3 \\
\hline $50-69$ & 89,5 & 99,2 & 33,5 & 98,3 & 5,3 & 97,5 & 90 & 10 \\
\hline$>69$ & 96,2 & 100 & 26,9 & 100 & 7,7 & 96,2 & 96,2 & 3,8 \\
\hline \multicolumn{9}{|l|}{ Escolaridade } \\
\hline Ensino fundamental & 91,7 & 100 & 29,2 & 91,7 & 16,7 & 95,8 & 79,2 & 20,8 \\
\hline Ensino médio & 87,5 & 96,7 & 30,3 & 94,1 & 9,2 & 94,7 & 86,2 & 13,8 \\
\hline Graduação & 86,6 & 99 & 36,9 & 96,4 & 4,8 & 96,2 & 86,8 & 13,2 \\
\hline Pós-graduação & 87,3 & 99,4 & 39,5 & 98,4 & 3,3 & 97,1 & 89 & 11 \\
\hline \multicolumn{9}{|l|}{ Renda } \\
\hline Até 1 salário mínimo & 90,9 & 100 & 34,1 & 93,2 & 10,2 & 95,5 & 92 & 8 \\
\hline 1 a 3 salários mínimos & 85 & 97,1 & 32,2 & 95,2 & 7,3 & 94,9 & 84,7 & 15,3 \\
\hline 3 a 6 salários mínimos & 88 & 99,5 & 38,7 & 98,2 & 3,3 & 97,2 & 86,8 & 13,2 \\
\hline 6 a 9 salários mínimos & 86,6 & 99,4 & 39 & 99,4 & 1,2 & 98,2 & 90,2 & 9,8 \\
\hline > 9 salários mínimos & 86,9 & 99,5 & 39,7 & 96,3 & 4,7 & 95,8 & 89,3 & 10,7 \\
\hline Nenhuma renda & 93,3 & 100 & 53,3 & 100 & 13,3 & 100 & 86,7 & 13,3 \\
\hline
\end{tabular}

Fonte: Autores (2020).

Dessa forma, observou-se que os entrevistados do sexo masculino tiveram um maior aumento percentual $16,4 \%$ em relação aos hábitos higiênicos do que a população feminina (10,4\%), porém o uso de máscara e o isolamento social foram mais adotados pelas mulheres, $91,8 \%$ e $89,9 \%$, respectivamente. Os entrevistados com maiores níveis de escolaridade foram os que apresentaram maiores modificações em suas condutas com o advento da pandemia. Houve aumento das práticas, de higienização das mãos dos entrevistados com graduação, 12,4\%, e pós-graduação, 12,1\%, e do uso de máscara, 91,4\% e $93,8 \%$, respectivamente.

Além disso, em relação ao medo da população rio-grandina em se infectar pelo novo vírus (medida numa escala numérica de 0 a 5, sendo zero ausência de medo e cinco medo extremo), os dados coletados mostraram houve uma maior porcentagem masculina, $4,4 \%(n=12)$, que relataram não ter medo de infecta-se pelo vírus, conforme Tabela 4 . 
Tabela 4 - Medo de infectar-se com o novo coronavírus (avaliado através de uma escala numérica crescente de 0 a 5 , sendo 0 ausência de medo e 5 medo extremo) dos entrevistados residentes na cidade de Rio Grande, Rio Grande do Sul, Brasil, desde o início da pandemia até o dia 30 de junho de 2020, de acordo com o sexo, faixa etária, escolaridade e renda.

\begin{tabular}{|c|c|c|c|c|c|c|c|c|c|c|c|c|}
\hline & \multicolumn{2}{|c|}{0} & \multicolumn{2}{|c|}{1} & \multicolumn{2}{|c|}{2} & \multicolumn{2}{|c|}{3} & \multicolumn{2}{|c|}{4} & \multicolumn{2}{|c|}{5} \\
\hline & $\mathbf{n}$ & $\%$ & $\mathbf{n}$ & $\%$ & $\mathbf{n}$ & $\%$ & $\mathbf{n}$ & $\%$ & $\mathbf{n}$ & $\%$ & $\mathbf{n}$ & $\%$ \\
\hline \multicolumn{13}{|l|}{ Sexo } \\
\hline Feminino & 16 & 1,7 & 35 & 3,8 & 87 & 9,5 & 259 & 28,3 & 234 & 25,6 & 284 & 31 \\
\hline Masculino & 12 & 4,4 & 21 & 7,7 & 29 & 10,6 & 81 & 29,7 & 71 & 26 & 59 & 21,6 \\
\hline \multicolumn{13}{|c|}{ Faixa etária (anos) } \\
\hline $18-29$ & 3 & 0,9 & 9 & 2,7 & 35 & 10,5 & 97 & 29,2 & 86 & 25,9 & 102 & 30,7 \\
\hline $30-49$ & 16 & 3,4 & 25 & 5,3 & 30 & 6,4 & 125 & 26,7 & 130 & 27,7 & 143 & 30,5 \\
\hline $50-69$ & 7 & 1,9 & 21 & 5,8 & 47 & 13 & 107 & 29,6 & 84 & 23,3 & 95 & 26,3 \\
\hline$>69$ & 2 & 7,7 & 1 & 3,8 & 4 & 15,4 & 11 & 42,3 & 5 & 19,2 & 3 & 11,5 \\
\hline
\end{tabular}

\begin{tabular}{|c|c|c|c|c|c|c|c|c|c|c|c|c|}
\hline Escolaridade & & & & & & & & & & & & \\
\hline Ensino fundamental & 2 & 8,3 & 2 & 8,3 & 2 & 8,3 & 6 & 25 & 2 & 8,3 & 10 & 41,7 \\
\hline Ensino médio & 8 & 5,3 & 10 & 6,6 & 23 & 15,1 & 40 & 26,3 & 31 & 20,4 & 40 & 26,3 \\
\hline Graduação & 10 & 6,6 & 24 & 4,8 & 46 & 9,2 & 143 & 28,5 & 125 & 25 & 153 & 30,5 \\
\hline Pós-graduação & 8 & 1,6 & 20 & 3,9 & 45 & 8,8 & 151 & 29,5 & 147 & 28,8 & 140 & 27,4 \\
\hline \multicolumn{13}{|l|}{ Renda } \\
\hline Até 1 salário mínimo & 4 & 4,5 & 2 & 2,3 & 10 & 11,4 & 22 & 25 & 11 & 12,5 & 39 & 44,3 \\
\hline 1 a 3 salários mínimos & 5 & 1,6 & 19 & 6,1 & 32 & 10,2 & 77 & 24,5 & 78 & 24,8 & 103 & 32,8 \\
\hline 3 a 6 salários mínimos & 11 & 2,8 & 18 & 4,6 & 34 & 8,7 & 120 & 30,5 & 104 & 26,5 & 106 & 27 \\
\hline 6 a 9 salários mínimos & 3 & 1,8 & 4 & 2,4 & 16 & 9,8 & 59 & 36 & 46 & 28 & 36 & 22 \\
\hline > 9 salários mínimos & 5 & 2,3 & 13 & 6,1 & 20 & 9,3 & 57 & 26,6 & 63 & 29,4 & 56 & 26,2 \\
\hline Nenhuma renda & 0 & 0 & 0 & 0 & 4 & 26,7 & 5 & 33,3 & 3 & 20 & 3 & 20 \\
\hline
\end{tabular}

Fonte: Autores (2020).

Também, foi observado que os indivíduos com a faixa etária entre 50 e 69 anos e acima de 69 anos tiveram maiores aumentos percentuais no que diz respeito ao uso de álcool em gel, 64,8 \% e 73,1\% respectivamente, por conseguinte, 96,2\% dos indivíduos acima de 69 anos afirmaram adotar o isolamento social (Tabela 3) enquanto os indivíduos entre 18 e 29 anos e 30 e 49 anos foram os que tiveram maior medo em infectar-se, 30,7 \% ( $\mathrm{n}=102)$ e 30,5\% ( $=143)$, respectivamente. Além 
disso, foi observado que indivíduos com ensino fundamental apresentaram níveis mais elevados de medo de infectar-se, $41,7 \%$ ( $n=10)$, em comparação aos entrevistados com maiores níveis de escolaridade (Tabela 4).

Os entrevistados com maiores níveis de escolaridade foram os que apresentaram maiores modificações em suas condutas com o advento da pandemia. Houve aumento das práticas, de higienização das mãos dos entrevistados com graduação, $12,4 \%$, e pós-graduação, $12,1 \%$, e do uso de máscara, $91,4 \%$ e $93,8 \%$, respectivamente (Tabela 3).

Também, foi observado que indivíduos com renda de seis a nove salários mínimos apresentaram um maior aumento em relação à lavagem das mãos com água e sabão, 12,8\%, e uso de máscara, 97\%, (Tabela 3). Já para a higienização das mãos com álcool o maior aumento observado, 63\%, foi entre os indivíduos com renda entre um e três salários mínimos (Tabela 3). Por fim, os indivíduos com renda de até um salário mínimo apresentaram maior adesão, $92 \%$, ao isolamento social (Tabela 3). Ademais, foi observado que indivíduos com as menores rendas apresentaram mais medo em infectar-se, de 44,3\% ( $\mathrm{n}=39$ ) para entrevistados com renda de até um salário mínimo e de 32,8\% $(\mathrm{n}=103)$ para entrevistados de um a três salários mínimos (Tabela 4).

\section{Discussão}

Desde o início da pandemia causada pelo vírus SARS-CoV-2 os esforços mundiais têm sido incessantes no que diz respeito a contenção da doença. Para isso, de acordo com especialistas, as medidas de proteção individual e isolamento social são ações cruciais diante da alta transmissibilidade da infecção (Rothan \& Byrareddy, 2020).

Segundo os estudos realizados por Lima et al. (2020) e Alsan et al. (2020), a população feminina demonstrou maior precaução frente à pandemia, já que se apresentaram mais receosas quanto ao risco de contaminação e aderiram mais às medidas profiláticas. Esses achados corroboram com o presente estudo, uma vez que, foi observado maior adesão feminina às medidas preventivas à COVID-19 e ao isolamento social, além de, as mulheres possuírem mais medo de infectar-se, quando comparada à população masculina. Isso pode ser explicado devido ao maior senso de autocuidado feminino, o que leva a maior adesão das medidas de prevenção por parte das mulheres. Dessa forma, essas ações são refletidas diretamente sobre número total de pessoas atingidas pela doença na cidade de Rio Grande, uma vez que as mulheres foram minoria em relação ao número de casos confirmados e ao número de óbitos, $47 \%$ e 37\%, respectivamente, até o dia 28 de novembro de 2020, segundo o Boletim Epidemiológico enunciado pela Secretaria Municipal da Saúde do Município de Rio Grande (SMS, 2020).

Em contrapartida, essa pesquisa apontou que apesar dos homens terem demonstrado maior mudança da frequência de lavagem das mãos, eles aderiram menos ao isolamento social, além de possuírem menos medo de infectar-se. Esses achados também são compatíveis com os estudos de Alsan et al. (2020) e Batista et al. (2020), realizados nos Estados Unidos e no Brasil respectivamente, em que indicaram a disparidade entre homens e mulheres no que diz respeito ao comportamento diante à pandemia. Essa diferença, pode ser atribuída ao fato de que, em um imaginário social, os homens são mais associados à invulnerabilidade e à força, características incompatíveis com a demonstração de autocuidado e medo (Gomes, 2012). Além de que, os indivíduos do sexo masculino são maioria no que diz respeito a população economicamente ativa na cidade de Rio Grande, o que pode contribuir para que esse grupo não consiga aderir ao isolamento social (IBGE, 2010a).

Apesar das atuais mudanças dessa ideia socialmente construída, sabe-se que os homens ainda se previnem menos às doenças em geral e procuram menos os serviços de saúde do que as mulheres, de forma que, sua atenção à saúde ocorre quando relacionados aos aspectos curativos (Laurenti, et al., 2005; Brito, et al., 2012). Isso pode influenciar, de maneira direta, uma maior taxa de mortalidade de homens por COVID-19, uma vez que essa população apresenta maiores índices de comorbidades, quando comparado às mulheres, contribuindo para um maior risco de óbito pelo novo coronavírus (Zhou et al., 2020). Visto isso, segundo o Boletim Epidemiológico disponibilizado pela Secretaria Municipal da Saúde do Município de Rio 
Grande (SMS), até o dia 28 de novembro de 2020, 63\% dos óbitos confirmados foram de indivíduos do sexo masculino, além de que a grande maioria já apresentava doenças prévias que poderiam afetar na evolução da COVID-19 (SMS, 2020).

Sabe-se que indivíduos de todas as faixas etárias podem ser infectados pelo novo coronavírus, porém, as manifestações e a gravidade da doença parecem ser mais exacerbadas quanto maior a idade e a presença de comorbidades, o que tornam os idosos o principal grupo de risco da COVID-19 (Cheng, et al., 2020). Nunes et al. (2020) avaliaram, com base nos dados obtidos pelo Estudo Longitudinal do Envelhecimento Brasileiro (ELSI-Brasil) de 2015 e 2016, a ocorrência de multimorbidade em indivíduos com 50 anos ou mais, fatores que são considerados condições de risco para a COVID-19 grave. Em seus resultados, os autores demonstraram que $80 \%$ da população entrevistada referiu ter pelo menos uma condição considerada agravante para a doença e 52\% apresentava multimorbidades. Esse cenário exalta a necessidade de uma atenção especial à população idosa uma vez que a idade e a comorbidade estão diretamente relacionadas com a evolução de doenças infecciosas, como a COVID-19 (Cheng, et al., 2020; Lenzi, et al., 2012).

Visto isso, Lima-Costa et al. (2020), a partir da iniciativa ELSI-COVID-19 - baseada em entrevistas telefônicas de participantes do ELSI-Brasil - investigaram, em uma primeira onda de inquéritos, a prevalência do comportamento preventivo da comunidade com 50 anos ou mais em 70 municípios brasileiros entre os dias 26 de maio e 8 de junho de 2020. Diante dos resultados, foram observadas altas prevalências $(97,3 \%)$ no que diz respeito à higienização das mãos e do uso de máscaras faciais ao sair de casa $(97,3 \%)$, além de que, indivíduos mais velhos foram mais propensos a permanecer em casa, se comparado aos mais jovens. Esses dados estão em consonância com os resultados obtidos pelo presente estudo, uma vez que os indivíduos acima de 50 anos tiveram maiores mudanças no que diz respeito ao uso de álcool em gel, além de que, quase a totalidade dos indivíduos acima de 69 anos afirmaram adotar o isolamento social.

Apesar das mudanças preventivas, 29,6\% ( $n=107)$ dos entrevistados entre 50 e 69 anos e 42,3\% (n=11) acima de 69 anos referiram ter medo intermediário, na escala numérica, em infectar-se (Tabela 4). Comparando-se às outras idades, esse achado é preocupante, uma vez que na cidade de Rio Grande, até o dia 28 de novembro de 2020, segundo a Prefeitura da Cidade de Rio Grande, 113 de 146 dos indivíduos que foram a óbito pelo vírus SARS-CoV-2 estão na faixa entre 60 e 89 anos. Por outro lado, até a mesma data, foi registrado que a maioria dos casos confirmados (3.406) no município, acometeram indivíduos entre 20 a 49 anos, em contraste, os resultados dessa pesquisa demonstraram que a comunidade com essa mesma faixa etária apresentou maiores níveis de medo em infectar-se. Esses resultados podem ser explicados por essa população ser considerada economicamente ativa, segundo o Departamento Intersindical de Estatísticas e Estudos Socioeconômicos (DIEESE) e, portanto, terem maior chance de se expor ao vírus e transmiti-lo, gerando mais medo (DIEESE, 2010) . Nesse sentido, por serem grandes veículos de transmissão, é indispensável reforçar a importância dos cuidados preventivos que a população jovem deve ter a fim de evitar a transmissão à população idosa, considerada a mais vulnerável à COVID-19.

Para mais, é fundamental destacar que pessoas com diferentes níveis socioeconômicos além serem expostas de maneira diferente ao risco de infecção, podem sofrer impactos de maneiras diferentes em diversas áreas de suas vidas. Dessa forma, indivíduos em condições sociais desfavoráveis, com menores rendas e níveis escolares baixos, estão mais susceptíveis a serem atingidas de forma mais marcante pela atual pandemia. Isso pode ser explicado pelo descumprimento das orientações em relação à prevenção da doença, devido à necessidade de sair de casa para trabalhar, utilizarem transporte público e terem menos acesso à recursos médicos (Lima et al.,2020).

A partir das análises feitas no presente trabalho, foi possível observar que os níveis mais altos de escolaridade e renda foram associados a maior adesão às medidas preventivas, como lavagem das mãos e uso de máscara. Alguns estudos apontaram dados semelhantes, como o realizado por de Souza et al. (2020). Esses autores observaram que municípios brasileiros em maior vulnerabilidade social apresentaram maiores taxas de incidência da doença. Lima et al. (2020), 
verificaram uma maior negligência quanto à pandemia pelos participantes com ensino fundamental, uma vez que aderiram menos ao isolamento social. Também, Machida et al. (2020) sugere que as famílias de baixa renda do Japão apresentaram menor adesão ao isolamento social e medidas de proteção individual. Em contrapartida, no presente estudo, os indivíduos com renda de um salário mínimo apresentaram maior porcentagem de isolamento social, 92\%. Talvez isso tenha relação com o medo de infectar-se que essa parcela populacional possui, em que quase a metade desses indivíduos afirmou ter medo extremo, conforme Tabela 4. Comportamento semelhante foi observado de acordo com as demais rendas, entretanto, não de forma tão expressiva quanto àqueles com baixa renda. Porém, é importante ressaltar que, dentre os entrevistados, os que possuem renda de até 1 salário mínimo são minoria $(\mathrm{n}=88)$ quando comparado ao número total de indivíduos da pesquisa $(\mathrm{n}=1.188)$, o que pode ser considerado como uma limitação desse estudo.

\section{Considerações Finais}

Apesar dos dados demonstrarem resultados positivos, no período do questionário, em relação às mudanças do comportamento em geral da população de Rio Grande frente à pandemia, é necessário destacar a importância de diferentes estratégias governamentais direcionadas aos diversos grupos sociais da cidade. Por meio desse estudo, foi possível observar que as diferenças socioeconômicas influenciam no comportamento dos cidadãos no que diz respeito aos cuidados com a doença e no medo em infectar-se.

Nesse sentido, é fundamental atentar-se para a população masculina, uma vez que fazem parte das estatísticas mais altas de casos confirmados e óbitos por COVID-19. Além disso, é imprescindível a realização de abordagens mais direcionadas aos jovens principalmente em relação às aglomerações, pois, apesar de os jovens não serem o grupo que mais sofre as complicações diretas da doença - comparando-se aos idosos -, eles atuam como vetores de transmissão e, além disso, quando são acometidos pelas formas graves da doença, contribuem para a sobrecarga dos sistemas de saúde, o que poderia ser evitado com medidas preventivas. Ademais, é necessário entender que os indivíduos menos favorecidos economicamente e com menores níveis de escolaridade sofrem os impactos da pandemia de formas diferentes, sendo indispensável um olhar mais atento do governo para essas camadas populares.

Nesse sentido, é pertinente destacar a importância da realização de estudos que abordem os aspectos sociais e econômicos da população em um contexto extremo, como por exemplo a pandemia causada pelo novo coronavírus. Assim, é possível haver melhor entendimento sobre os anseios populacionais, e, a partir dessa compreensão, readequar as campanhas e condutas aos diferentes grupos populacionais. Há necessidade de realizar novos estudos acerca da influência dos hábitos comportamentais das pessoas em relação à propagação da pandemia visando a aprimorar a abordagem das políticas públicas aos grupos mais vulneráveis. Isso corrobora para encurtar barreiras invisíveis entre interlocutor e expectador ao passo em que as informações são simplificadas e direcionadas aos públicos específicos, contribuindo para uma maior adesão e efetividade dessas campanhas de conscientização.

\section{Referências}

Alsan, M., Stantcheva, S., Yang, D., \& Cutler, D. (2020). Disparities in coronavirus 2019 reported incidence, knowledge, and behavior among US adults. JAMA network open, 3(6), e2012403-e2012403. 10.1001/jamanetworkopen.2020.12403

Aquino, E. M., Silveira, I. H., Pescarini, J. M., Aquino, R., \& Souza-Filho, J. A. D. (2020). Medidas de distanciamento social no controle da pandemia de COVID-19: potenciais impactos e desafios no Brasil. Ciência \& Saúde Coletiva, 25, 2423-2446, https://doi.org/10.1590/1413-81232020256.1.10502020

Batista, S. R., Souza, A. S. S. D., Nogueira, J., Andrade, F. B. D., Thumé, E., Teixeira, D. S. D. C., ... \& Nunes, B. P. (2020). Comportamentos de proteção contra COVID-19 entre adultos e idosos brasileiros que vivem com multimorbidade: iniciativa ELSI-COVID-19. Cadernos de Saúde Pública, 36, e00196120, https://doi.org/10.1590/0102-311x00196120. 
Brito, Annie Mehes Maldonado, \& Camargo, Brigido Vizeu. (2011). Representações sociais, crenças e comportamentos de saúde: um estudo comparativo entre homens e mulheres. Temas em Psicologia, 19(1), 283-303. http://pepsic.bvsalud.org/scielo.php?script=sci_arttext\&pid=S1413$389 \mathrm{X} 2011000100023 \& \operatorname{lng}=\mathrm{es} \& \operatorname{lng}=\mathrm{pt}$.

Centro Regional de Estudos para o Desenvolvimento da Sociedade da Informação (CETIC). Tic domicílios, 2018. https://data.cetic.br/cetic/explore?idPesquisa=TIC_DOM\&idUnidadeAnalise=Usuarios\&ano=2018

Cheng, Z. J. \& Shan J. (2020). Novel coronavirus: where we are and what we know. Infection, 48(2), 155-163, 10.1007/s15010-020-01401-y

Cowling, B. J., Chan, K. H., Fang, V. J., Cheng, C. K., Fung, R. O., Wai, W., \& Chiu, B. C. (2009). Facemasks and hand hygiene to prevent influenza transmission in households: a cluster randomized trial. Annals of internal medicine, 151(7), 437-446, 10.7326/0003-4819-151-7-200910060-00142

de Souza, C.D.F., Machado, M.F. \& do Carmo, R.F. Human development, social vulnerability and COVID-19 in Brazil: a study of the social determinants of health. Infect Dis Poverty 9, 124 (2020). https://doi.org/10.1186/s40249-020-00743-x

Decreto ${ }^{\circ}$ 17.045, de 19 de Março de 2020, Município de Rio Grande - RS. Ações durante a pandemia de COVID-19 - Estado de Emergência no Município do Rio Grande (2020). https://www.riogrande.rs.gov.br/pagina/publicacao/decreto-17-045-estado-de-emergencia-no-municipio-do-rio-grande/

Departamento Intersindical de Estatísticas e Estudos Socioeconômicos (DIEESE) (2010). Estimativa da população economicamente ativa por faixa etária Observatório Nacional da Economia Solidária e do Cooperativismo. https://ecosol.dieese.org.br/ws2/tabela/2311

Ferguson, N., Laydon, D., Nedjati Gilani, G., Imai, N., Ainslie, K., Baguelin, M., \& Dighe, A. (2020). Impact of non-pharmaceutical interventions (NPIs) to reduce COVID-19 mortality and healthcare demand. Imperial College London. https://doi.org/10.25561/77482

Ferreira, L. B. \& Ramos, D. F. (2020). Remdesivir e lopinavir/ritonavir frente ao novo coronavírus: breve revisão. VITTALLE-Revista de Ciências da Saúde. 32(1), 43-55. https://doi.org/10.14295/vittalle.v32i1.11572

Figueiredo, W (2005). Assistência à saúde dos homens: um desafio para os serviços de atenção primária. Ciência \& Saúde Coletiva, 10(1), 105-109. https://doi.org/10.1590/S1413-81232005000100017

Gomes, R., Nascimento, E. F. D., \& Araújo, F. C. D. (2007). Por que os homens buscam menos os serviços de saúde do que as mulheres? As explicações de homens com baixa escolaridade e homens com ensino superior. Cadernos de Saúde Pública, 23, 565-574. https://doi.org/10.1590/S0102-311X2007000300015

Guimarães, R. (2020). Anti-Covid vaccines: a look from the Collective Health. Ciência \& saúde coletiva, 25, 3579-3585. https://doi.org/10.1590/141381232020259.24542020

Instituto Brasileiro de Geografia e Estatística (IBGE) (2010a). Estatística de Gênero. População economicamente ativa. https://www.ibge.gov.br/apps/snig/v1/?loc=0,431560\&cat=-1,1,2,-2,-,128\&ind=4726

Instituto Brasileiro de Geografia e Estatística (IBGE) (2010b). Sinopse do censo demográfico 2010. https://censo2010.ibge.gov.br/sinopse/index.php

Laurenti, R., Mello-Jorge, M. H. P. \& Gotlieb S. L. D. (2005). Perfil epidemiológico da morbi-mortalidade masculina. Ciência Saúde Coletiva, 10(1), 35-46. https://doi.org/10.1590/S1413-81232005000100010

Lenzi, L., Mello, A. M. D., Silva, L. R. D., Grochocki, M. H. C., \& Pontarolo, R. (2012). Influenza pandêmica A (H1N1) 2009: fatores de risco para o internamento. Jornal Brasileiro de Pneumologia, 38(1), 57-65. https://doi.org/10.1590/S1806-37132012000100009

Lima, D. L. F., Dias, A. A., Rabelo, R. S., Cruz, I. D. D., Costa, S. C., Nigri, F. M. N., \& Neri, J. R. (2020). COVID-19 no estado do Ceará, Brasil: comportamentos e crenças na chegada da pandemia. Ciência \& Saúde Coletiva, 25, 1575-1586. https://doi.org/10.1590/1413-81232020255.07192020

Lima-Costa, M. F., Mambrini, J. V. D. M., Andrade, F. B. D., Peixoto, S. W. V., \& Macinko, J. (2020). Social distancing, use of face masks and hand washing among participants in the Brazilian Longitudinal Study of Aging: the ELSI-COVID-19 initiative. Cadernos de Saúde Pública, 36. https://doi.org/10.1590/0102-311x00193920

Machida, M., Nakamura, I., Saito, R., Nakaya, T., Hanibuchi, T., Takamiya, T., \& Kojima, T. (2020). Changes in implementation of personal protective measures by ordinary Japanese citizens: A longitudinal study from the early phase to the community transmission phase of the COVID-19 outbreak. International Journal of Infectious Diseases, 96, 371-375. 10.1016/j.ijid.2020.05.039

Mbakaya, B. C., Lee, P. H. \& Lee, R. L. (2017). Hand hygiene intervention strategies to reduce diarrhoea and respiratory infections among schoolchildren in developing countries: a systematic review. International journal of environmental research and public health, 14(4), 371. 10.3390/ijerph14040371

Nunes, B. P., Souza, A. S. S. D., Nogueira, J., Andrade, F. B. D., Thumé, E., Teixeira, D. S. D. C. \& Batista, S. R. (2020). Multimorbidity and population at risk for severe COVID-19 in the Brazilian Longitudinal Study of Aging. Cadernos de Saúde Pública, 36(12). https://doi.org/10.1590/0102-311x00129620

Nussbaumer-Streit, B., Mayr, V., Dobrescu, A. I., Chapman, A., Persad, E., Klerings, I., \& Gartlehner, G. (2020). Quarantine alone or in combination with other public health measures to control COVID-19: a rapid review. Cochrane Database of Systematic Reviews. https://doi.org/10.1002/14651858.CD013574

Organização Mundial da Saúde, OMS. (2020). Uso racional de equipamento de proteção individual (EPI) para doença coronavírus (COVID-19): orientação provisória. https://apps.who.int/iris/handle/10665/331498

Organização Pan-Americana da Saúde, OPAS. (2020). Folha informativa - COVID-19 (doença causada pelo novo coronavírus). https://www.paho.org/bra/index.php?option=com_content \&view=article\&id=6101:covid19\&Itemid=875.

Pereira A.S.; Shitsuka D. M.; Parreira F. J.; Shitsuka R. (2018). Metodologia da pesquisa científica. [e-book]. Santa Maria. Ed. UAB/NTE/UFSM. em: https://repositorio.ufsm.br/bitstream/handle/1/15824/Lic_Computacao_Metodologia-Pesquisa-Cientifica.pdf?sequence=1 
Research, Society and Development, v. 10, n. 2, e29010212413, 2021

(CC BY 4.0) | ISSN 2525-3409 | DOI: http://dx.doi.org/10.33448/rsd-v10i2.12413

Pestana, J. M., \& Pestana, R. C. (2020). The face mask as an established symbol against COVID-19. Revista da Associação Médica Brasileira, 66(10), 13181319. https://doi.org/10.1590/1806-9282.66.10.1318

Rio Grande, RS. (2020). Modelo de Distanciamento Controlado Rio Grande Do Sul. https://distanciamentocontrolado.rs.gov.br/

Rodriguez-Morales A. J., Gallego V., Eescalera-Antezana J. P., Méndez C. A., Zambrano L. I., Franco-Paredes C., Suárez J. A., Rodriguez-Enciso H. D., Balbin-Ramon G. J., Savio-Larriera E., Risquez A. \& Cimerman S. (2020). COVID-19 in Latin America: The implications of the first confirmed case in Brazil. Travel Medicine and Infectious Disease, 35: 101613. 10.1016/j.tmaid.2020.101613

Rothan, H. A., \& Byrareddy, S. N. (2020). The epidemiology and pathogenesis of coronavirus disease (COVID-19) outbreak. Journal of autoimmunity, 109, 102433. https://doi.org/10.1016/j.jaut.2020.102433

Secretaria Estadual da Saúde do Estado do Rio Grande do Sul, SES-RS. (2020). Decretos Estaduais. https://coronavirus.rs.gov.br/decretos-estaduais

Secretaria Estadual da Saúde do Estado do Rio Grande do Sul, SES-RS. (2021). Painel Coronavírus. https://ti.saude.rs.gov.br/covid19/

Secretaria Municipal da Saúde do Município de Rio Grande (SMS) (2020). Ações durante a pandemia de COVID-19. BOLETIM EPIDEMIOLÓGICO, Semana epidemiológica 48 - 22/11 até 28/11 de 2020. https://www.riogrande.rs.gov.br/pagina/wp-content/uploads/2020/11/SEMANA48_BOLETIMEPIDEMIOLOGICO.pdf

Shereen M. A., Khan S., Kazmi A., Bashir N., Siddique R. (2020). COVID-19 infection: origin, transmission, and characteristics of human coronaviruses. Journal of Advanced Research, 24, 91-98. https://doi.org/10.1016/j.jare.2020.03.005

West, R., Michie, S., Rubin, G. J., \& Amlôt, R. (2020). Applying principles of behaviour change to reduce SARS-CoV-2 transmission. Nature Human Behaviour,4, 451-459. https://doi.org/10.1038/s41562-020-0887-9

World Health Organization - WHO. (2020a). Coronavirus disease (COVID-19) advice for the public. https://www.who.int/emergencies/diseases/novelcoronavirus-2019/advice-for-public.

World Health Organization - WHO. (2020b). WHO Director-General's opening remarks at the media briefing on COVID-19 - 11 March 2020. https://www.who.int/director-general/speeches/detail/who-director-general-s-opening-remarks-at-the-media-briefing-on-covid-19---11-march-2020.

Zhou F., Yu T., Du R., Fan G., Liu Y. \& Cao B. (2020). Clinical course and risk factors for mortality of adult inpatients with COVID-19 in Wuhan, China: a retrospective cohort study. Lancet, 395, 1054-1062. https://doi.org/10.1016/S0140-6736(20)30566-3 